\title{
Spark Ignition of Propane-Air Mixtures Near the Minimum Ignition Energy: Part II. A Model Development
}

\author{
Y. KO and V. S. ARPACI \\ Department of Mechanical Engineering and Applied Mechanics, University of Michigan, Ann Arbor, MI \\ 48109 \\ and
}

R. W. ANDERSON

Engine Research Department, Research Staff, Ford Motor Company, Dearborn, MI 48121-2053

\begin{abstract}
A model is developed to simulate the kernel growth observed in the exprimental study of Part I. Kernel growth, described as a two-step process, initially involves a blast wave over a negligible short time followed by a diffusive growth with an electrical input power. The diffusive growth is formulated by an integral approach involving temperature dependent overall reaction kinetics and electrode heat loss. The model predicts the kernel growth reasonably well with the measured spark power input. It predicts both ignition and nonignition kernel growth. The existence of a critical radius is also demonstrated. In addition, dimensional analyses are given to clarify the physical aspects of the critical radius and the characteristic radius of the blast wave.
\end{abstract}

\section{NOMENCLATURE}

$A \quad$ fuel or limiting reactant

a dimensionless mass fraction of $A(=$ $\left.Y_{A} / Y_{A_{u}}\right)$

$B_{A} \quad$ frequency factor

$c \quad$ speed of sound

$C_{d} \quad$ constant for discharge induced entrainment flow

$C_{i} \quad$ nonlinear coefficients $(i=1, \ldots, 11$, $A, e)$

$C_{p(v)}$

$D$

d

$D_{A} \quad$ binary diffusion coefficient of $A$ into mixture

$E_{d} \quad$ measured spark discharge energy

$\dot{E}_{e} \quad$ electrical discharge input power
$E_{f} \quad$ discharge energy into the fall region

$E_{G}, \dot{E}_{G}$ spark energy and power remaining in the gas

$E_{p}, \dot{E}_{p}$ discharge energy and power into the positive column

$E_{o} \quad$ instantaneous energy release per unit length

Fo $\quad$ Fourier number $\left(=\alpha t / L^{2}\right)$

$h \quad$ enthalpy of mixture

$I_{s} \quad$ measured secondary current

$k$ thermal conductivity

$L \quad$ characteristic length

$l \quad$ length of cylindrical blast

$l_{a}$ planar adiabatic flame thickness (= $\left.k / \rho_{u} C_{p} U_{a}\right)$

$\mathrm{Le}_{A} \quad$ Lewis number $\left(=k / \rho C_{p} D_{A}\right)$

$M \quad$ gas flow Mach number $(u / c)$

$m \quad$ mass in the kernel 


\begin{tabular}{|c|c|}
\hline $\begin{array}{l}\dot{m} \\
\dot{m}_{e}\end{array}$ & $\begin{array}{l}\text { mass flow rate of mixture per unit area } \\
\text { entrainment mass flow rate due to elec- } \\
\text { trical discharge }\end{array}$ \\
\hline $\boldsymbol{P}$ & pressure \\
\hline$q_{r_{f}}$ & $\begin{array}{l}\text { rate of heat conduction per unit area at } \\
r=r_{f}\end{array}$ \\
\hline $\begin{array}{l}Q_{A} \\
R\end{array}$ & $\begin{array}{l}\text { heat of combustion per unit mass of } A \\
\text { dimensionless radius or spatial coordi- } \\
\text { nate }\end{array}$ \\
\hline$r$ & radius or spatial coordinate \\
\hline$R_{c}$ & dimensionless critical radius \\
\hline$r_{c}$ & critical radius \\
\hline$r_{0}$ & $\begin{array}{l}\text { characteristic radius of cylindrical blast } \\
\text { wave }\end{array}$ \\
\hline $\boldsymbol{R}$ & dimensionless radius $\left(=r / l_{a}\right)$ \\
\hline$T$ & temperature \\
\hline$t$ & time or time coordinate \\
\hline$t_{d}$ & discharge duration \\
\hline$\dot{U}$ & laminar burning velocity \\
\hline$u$ & gas flow velocity \\
\hline$u_{e}$ & $\begin{array}{l}\text { representative discharge induced en- } \\
\text { trainment velocity }\end{array}$ \\
\hline $\boldsymbol{V}$ & volume of the kernel \\
\hline$x$ & space \\
\hline$Y_{A}$ & mass fraction of $A$ \\
\hline
\end{tabular}

$\begin{array}{ll}\lambda & \text { dimensionless radiv }\left(=r / r_{o}\right) \\ \rho & \text { density } \\ \tau & \text { dimensionless im }\left(c_{o} t / r_{o}\right) \\ \phi & \text { equivalence ra } r \\ \omega_{A} & \text { chemical reach a rate of } A \text { per unit } \\ & \text { volume }\end{array}$

\section{Special Symbols}

$\begin{array}{ll}\mathscr{E} & \text { activation energy } \\ \mathscr{H} & \text { molecular weight of the gas } \\ \Re & \text { universal gas constant } \\ \mathscr{R} & \text { gas constant }(=\Re / \mathscr{K})\end{array}$

\section{Subscripts}

$\begin{array}{ll}a & \text { adiabatic flame } \\ b & \text { burned gas of steady spherical flame } \\ c & \text { critical value } \\ f & \text { flame kernel } \\ u & \text { unburned fresh mixture } \\ o & \text { ambient gas }\end{array}$

\section{Superscripts}

\section{$A$ fuel or limiting reaction $A$}

\section{Greek Symbols}

$\alpha \quad$ thermal diffusivity

$\beta \quad$ reduced activation energy $\left(=\mathscr{E}\left(T_{a}-\right.\right.$ $\left.T_{u}\right) /\left(\Re T_{b}^{2}\right)$ )

$\gamma \quad$ specific heat ratio

$\boldsymbol{\delta}_{\boldsymbol{A}} \quad$ fuel penetration depth

$\delta_{T} \quad$ heating zone thickness (thermal penetration depth)

$\epsilon \quad$ reduced heat of combustion $\left(=\left(T_{a}-\right.\right.$ $\left.\left.T_{u}\right)\left(T_{b}\right)\right)$

$\zeta$ dimensionless spatial coordinate in reaction zone

$\eta_{d}(t) \quad$ instantaneous discharge efficiency

$\eta_{d}\left(t_{d}\right)$ average discharge efficiency over discharge duration $t_{d}$

$\eta_{d}^{*} \quad E_{G} / E_{p}$ in Table 4

$\eta_{g} \quad$ discharge efficiency due to quenching

$\theta$ dimensionless temperature $(=(T-$ $\left.\left.T_{u}\right) /\left(T_{a}-T_{u}\right)\right)$

$\theta_{1}$ dimensionless temperature in Eq. 10

$\Lambda \quad$ eigenvalue of planar premixed flame

\section{INTRODUCTION}

Spark ignition by a time-dependent electrical discharge through electrodes is an unsteady heat and mass transfer process involving chemical reactions. Modeling this process requires an understanding of the breakdown process, the electrical discharge and the effect of electrodes, in addition to gas dynamics and chemical kinetics. The main objective of the present study is to develop an intuitive ignition model and improve understanding of spark induced flame initiation. The model is validated by the experimental data obtained in Part I [1].

There is an extensive literature on modeling of spark ignition [2-27]. Only a few models for spark kernel growth, however, are validated with experimental data $[7-9,11,13,23]$. Also, the shock wave associated with breakdown and the effect of electrodes do not appear to be taken into account in most of the present models, though 
considering a momentum equation enables to characterize the shock wave in a very complex manner [14, 17, 24-27]. Only, Lim et al. [7, 8], Ko [9], and Adelman [11] use the blast wave concept to characterize the shock wave associated with breakdown. Lim et al. [7, 8] and Ko [9] also account for fall energy losses.

Flame initiation in a premixed mixture by an electric spark may be modeled as a two-step process; (1) flame formation and (2) flame kernel development [16]. Flame formation is always ensured with a successful breakdown because a high-temperature kernel is generated after breakdown [28, 29]. After this formation, thermal diffusion dominates flame kernel development $[7-9,16,30-32]$. The development is successful when the power input from the spark is sufficient to drive the flame kernel beyond the critical size $[1,9,13,15,31-35]$. The above two-step process can be identified by a short period of blast wave followed by a relatively long diffusive growth $[7,8]$. The blast wave is evidenced by a shock front that rapidly decays to an acoustic wave in a few microseconds, which in turn is followed by a growing diffusion boundary layer. In the present study, the diffusive growth coupled with the blast wave model of $\mathrm{Lim}$ et al. $[7,8]$ is formulated by an integral approach involving simple overall Arrhenius reaction kinetics. A time-dependent electrical discharge is considered in addition to the effects of electrodes and discharge characteristics on the ignition process.

The study consists of seven sections: following this introduction, Section 2 presents dimensional arguments of a critical spherical flame, Sec. 3 outline kernel growth, Sec. 4 presents the diffusion model, Sec. 5 compares model predictions with experimental data and discusses the predictions, and Sec. 6 summarizes and concludes the study.

\section{CRITICAL SPHERICAL FLAME- DIMENSIONAL ARGUMENTS}

Consider an unsupported, convection-free, spherical, lean premixed flame in infinite space. Diffusional processes feed the reactant into the reaction zone at such a rate that the heat generation bal- ances the conduction loss to the unburned mixture and a steady flame is maintained. The usual conservation equations for energy and a limiting reactant $A$ of the steady flame are

$$
\begin{aligned}
& \frac{1}{r^{2}} \frac{d}{d r}\left(r^{2} k \frac{d T}{d r}\right)+Q_{A} \omega_{A}=0, \\
& \frac{1}{r^{2}} \frac{d}{d r}\left(r^{2} \rho D_{A} \frac{d Y_{A}}{d r}\right)-\omega_{A}=0,
\end{aligned}
$$

where the chemical reaction rate of $A$ per unit volume is

$\omega_{A}=\rho^{2} B_{A} Y_{A} \exp \left(-\frac{\mathscr{E}}{\Re T}\right)$.

Here $T, Y_{A}$, and $r$ denote temperature, mass fraction of fuel $A$, and radius, respectively, $\rho$, $k$, and $D_{A}$ are density, thermal conductivity, and binary diffusion coefficient of $A$ into the mixture, respectively, $Q_{A}, \Re$, and $\mathscr{E}$ denote heat of combustion per unit mass of $A$ (lower heating value), universal gas constant, and activation energy, respectively, and $B_{A}$ is a frequency factor. The boundary conditions are

$$
\begin{aligned}
& r \rightarrow \infty: T=T_{u}, Y_{A}=Y_{A_{u}}, \\
& r \rightarrow 0: \frac{d T}{d r}=0, \frac{d Y_{A}}{d r}=0 .
\end{aligned}
$$

The subscript $u$ corresponds to the unburned fresh mixture.

Combining Eqs. 1 and 2, and integrating the resulting equation subject to Eq. 3 readily gives the steady solution $[15,31,32]$

$$
\frac{T-T_{u}}{T_{a}-T_{u}}+\frac{1}{\operatorname{Le}_{A}} \frac{Y_{A}}{Y_{A_{u}}}=\frac{1}{\operatorname{Le}_{A}},
$$

where $\operatorname{Le}_{A}=k / \rho C_{p} D_{A}$ is the Lewis number, with $C_{p}$ being the specific heat at a constant pressure, and $T_{a}=T_{u}+Q_{A} Y_{A_{u}} / C_{p}$ is the adiabatic flame temperature. Because $Y_{A} \approx 0$ in the burned gas, the burned gas temperature $T_{b}$ obtained from Eq. 4 is

$T_{b}=T_{u}+\frac{1}{\operatorname{Le}_{A}}\left(T_{a}-T_{u}\right)$. 
For $\operatorname{Le}_{A}>1$, the burned gas temperature of the steady flame is lower than the adiabatic flame temperature, i.e., $T_{b}<T_{a}$, while $T_{b}>T_{a}$ for $\mathrm{Le}_{A}<1$.

Nondimensionalization of Eqs. 1 and 4 yields

$$
\begin{aligned}
-\frac{1}{R^{2}} & \frac{d}{d R}\left(R^{2} \frac{d \theta}{d R}\right) \\
& =\beta^{2} \Lambda a \exp \left(\frac{\beta\left(\theta-\theta_{b}\right)}{1+\epsilon\left(\theta-\theta_{b}\right)}\right),
\end{aligned}
$$

$\theta+\frac{a}{\mathrm{Le}_{A}}=\frac{1}{\mathrm{Le}_{A}}$,

where $\theta=\left(T-T_{u}\right) /\left(T_{a}-T_{u}\right), \quad a=Y_{A} / Y_{A_{u}}$, $R=r / l_{a}, l_{a}=k / \rho_{u} C_{p} U_{a}$, being the adiabatic planar flame thickness, where $U_{a}$ is the adiabatic burning velocity, $\epsilon=\left(T_{a}-T_{u}\right) / T_{b}, \beta=\mathscr{E}\left(T_{a}\right.$ $\left.-T_{u}\right) / \Re T_{b}^{2}, \theta_{b}=1 / L_{A}$, and $\Lambda$ is the eigenvalue of the planar premixed flame,

$\Lambda=\frac{1}{\beta^{2}} \frac{l_{a}^{2}}{k} C_{p} \rho^{2} B_{A} \exp \left(-\frac{\mathscr{E}}{\Re T_{b}}\right)$.

With the assumption of large activation energy $(\beta \gg 1)$, the reaction rate becomes strongly dependent upon temperature and the reaction is frozen except in a zone that is much thinner than either that of the heating zone or the dimensionless radius $R_{c}$ of the steady flame. Then, the solutions of Eqs 6 and 7 satisfying Eq. 3, for $R<R_{c}$,

$$
\theta=\theta_{b}=\frac{1}{\operatorname{Le}_{A}}, \quad a=0,
$$$$
\left(T=T_{b}, Y_{A}=0\right) \text {, }
$$

and, for $R>R_{c}$,

$$
\begin{aligned}
& \theta=\frac{R_{c}}{\operatorname{Le}_{A} R}, \quad a=1-\frac{R_{c}}{R} \\
& \left(\frac{T-T_{u}}{T_{a}-T_{u}}=\frac{1}{\operatorname{Le}_{A}} \frac{r_{c}}{r}, Y_{A}=Y_{A_{u}}\left(1-\frac{r_{c}}{r}\right)\right) .
\end{aligned}
$$

However, in the thin reaction zone, the effect of reaction needs to be taken into account. As $\beta \rightarrow \infty$, the dimensionless temperature within the zone becomes

$\theta=\frac{1}{\mathrm{Le}_{A}}+\frac{\theta_{1}}{\beta}+O\left(\frac{1}{\beta^{2}}\right), \quad \theta_{1}=O(1)$,

and the dimensionless radius of the zone as

$R=R_{c}+\frac{\zeta}{\beta}, \quad \zeta=O(1)$,

where $\theta_{1} / \beta$ and $\zeta / \beta$ are, respectively, the dimensionless temperature and space variation within the reaction zone. Because the curvature is negligible in the thin reaction zone, Eq. 6 reduces to

$-\frac{d^{2} \theta}{d R^{2}}=\beta^{2} \Lambda a \exp \theta_{1}$,

or, in terms of $d \theta=d \theta_{1} / \beta$ and $d R=d \zeta / \beta$,

$-\frac{d^{2} \theta_{1}}{d \zeta^{2}}=\beta \Lambda a \exp \theta_{1}$.

Following dimensional arguments clarify the physical aspects of the dimensionless radius $R_{c}$. From Eqs. 7 and 10, $a \sim \operatorname{Le}_{A} \theta_{1} / \beta$, and Eq. 12 gives

$\frac{\theta_{1}}{\zeta^{2}} \sim \Lambda \mathrm{Le}_{A} \theta_{1}, \quad$ or $\quad \zeta^{2} \Lambda \mathrm{Le}_{A} \sim 1$

Separately, from Eqs. 9 and 10,

$\theta=\frac{R_{c}}{\operatorname{Le}_{A} R}=\frac{1}{\operatorname{Le}_{A}\left(1+\zeta / \beta R_{c}\right)}$,

which, in view of $\zeta / \beta R_{c} \ll 1$,

$\theta=\frac{1}{\operatorname{Le}_{A}}\left(1-\frac{\zeta}{\beta R_{c}}\right)=\frac{1}{\operatorname{Le}_{A}}-\frac{\zeta}{\operatorname{Le}_{A} \beta R_{c}}$.

A comparison between Eqs. 10 and 14 implies

$\theta_{1} \sim O(1) \sim \frac{\zeta}{\operatorname{Le}_{A} R_{c}}, \quad$ or $\quad \zeta \sim \operatorname{Le}_{A} R_{c}$. 
Finally, combination of the Eqs. 13 and 15 results in a dimensionless radius of the steady spherical flame,

$$
R_{c} \sim \frac{1}{\left(\Lambda \operatorname{Le}_{A}{ }^{3}\right)^{1 / 2}},
$$

which, within a constant, is the result obtained by Champion et al. [15] following a considerably involved asymptotic matching,

$$
R_{c}=\frac{1}{\left(2 \Lambda \operatorname{Le}_{A}^{3}\right)^{1 / 2}} \text {. }
$$

Now, in terms of $\Lambda$ from [36] and assuming $k$ $\sim \sqrt{T}$ and $B_{A} \sim \sqrt{T}$ [37], the dimensional radius of the steady flame $r_{c}$ becomes [15]

$$
\begin{aligned}
r_{c}= & \frac{(k)_{T_{a}}}{\left(C_{p}\right)_{T_{a}}(\rho)_{T_{u}} U_{a}} \frac{T_{a}}{T_{b} \mathrm{Le}_{A}} \\
& \times \exp \left(\frac{\mathscr{E}}{2 \Re}\left(\frac{1}{T_{b}}-\frac{1}{T_{a}}\right)\right) .
\end{aligned}
$$

The radius is very sensitive to the diffusivity of the limiting reactant of the chemical process. Because of the effect of the Lewis number on the burned gas temperature $T_{b}$ of the steady flame (Eq. 5), the radius increases with a larger Lewis number.

Small perturbation stability analysis of the steady spherical flame for steady radius $[15,31$, 32] leads to $r_{f}=r_{c}$ for the marginal state, $r_{c}$ implying the critical radius. Successful flame initiation requires that the flame grow beyond $r_{c}$ $[15,31,32]$. Experimental observations on spark ignition in a propane-air mixture support this point of view $[1,9,15,34]$. As expected from the nature of the critical flame, the flame speed with a slightly supercritical power input decreases as the radius of the flame kernel approaches $r_{c}$ while soon after as the kernel grows larger than $r_{c}$ the flame speed increases and approaches the adiabatic flame speed [15]. A similar trend of flame kernel growth is also observed in an engine for low-speed operation [38].

\section{KERNEL GROWTH}

Experimental observations indicate that kernel growth can be described as a two-step process. The early part of growth is thermomechanical (waves) and the later part is a thermal (diffusion) penetration. The thermomechanical wave is a blast wave that starts as a shock wave $(M>1)$ but rapidly decays to an acoustic wave $(0<M<1)$, $\boldsymbol{M}$ being the Mach number.

The fundamental difference between a blast wave and a diffusive penetration is that the former is momentum-controlled while the latter is thermal energy-controlled. A dimensionless number that characterizes the importance of a blast wave relative to diffusive penetration can be derived from the ratio of kinetic energy and thermal conduction:

$$
\frac{\partial\left(\rho u^{2}\right)}{\partial t} / \frac{\partial}{\partial x}\left(k \frac{\partial T}{\partial x}\right)
$$

where $u, x$, and $t$, respectively, denote velocity, space, and time. On dimensional grounds,

$\frac{\rho u^{2}}{t} / \frac{k T}{L^{2}} \sim \frac{\rho u^{2} L^{2}}{t k T}$,

which can be rearranged in terms of

$c^{2}=\gamma \frac{P}{\rho}=\gamma \Re T, \quad \frac{\Re}{C_{p}}=\frac{\gamma-1}{\gamma}, \quad \frac{k}{\rho C_{p}}=\alpha$

as

$(\gamma-1)\left(\frac{u^{2}}{c^{2}}\right) \frac{L^{2}}{\alpha t} \sim(\gamma-1) \frac{M^{2}}{\text { Fo }}$

where $\alpha, \gamma, c$, and $L$, respectively, denote thermal diffusivity, specific heat ratio, isentropic speed of sound, and a characteristic length, and Fo $=\alpha t / L^{2}$ is the Fourier number. At the transition, kinetic energy and diffusion are equally important and

$$
\frac{(\gamma-1) M^{2}}{\text { Fo }} \sim 1,
$$


which leads, in view of $u=L / t$, to

$t \sim\left[\frac{(\gamma-1) L^{4}}{\alpha c^{2}}\right]^{1 / 3}$

For a typical spark discharge in automotive engine use, for example, the initial energy input is about $1 \mathrm{~mJ}$ and the kernel size is on the order of 1 $\mathrm{mm}$. For a kernel temperature of $2000 \mathrm{~K}$,

$t \sim\left[\frac{(1.4-1)\left(10^{-3}\right)^{4}}{\left(2 \times 10^{-4}\right)(850)^{2}}\right]^{1 / 3} \sim 10^{-5} \mathrm{~s}$,

which implies that the transition from a blast wave to thermal diffusion occurs on the order of microseconds after spark initiation.

In a manner similar to the foregoing dimensional consideration for $t$, a radius $r_{o}$ characterizing the cylindrical blast wave due to an instantaneous energy release per unit length, $E_{o}$ can be found as follows. Assuming that the original energy release is transformed into kinetic energy,

$E_{o} l \sim \rho_{o} c_{o}{ }^{2} r_{o}{ }^{2} l$,

which, for an isentropic flow, can be rearranged in terms of $c_{o}{ }^{2}=\gamma \Re T_{o}=\gamma P_{o} / \rho_{o}$ as

$$
\frac{E_{o}}{r_{o}^{2}} \sim \gamma P_{o}, \quad \text { or } \quad r_{o} \sim\left(\frac{E_{o}}{\gamma P_{o}}\right)^{1 / 2} \text {, }
$$

which, within a constant, is the characteristic length normally used for nondimensionalization in the literature $[39,40]$. Following a computational study, Jones [41] finds

$r_{o}=\left(\frac{E_{o}}{B \gamma P_{o}}\right)^{1 / 2}$,

where $B=3.94$ for $\gamma=1.4$. Here $P_{o}, \rho_{o}$, and $c_{o}$ are the pressure, density, and isentropic speed of sound of the ambient gas, respectively, and $l$ is a length of the cylindrical blast. This wave can be described with a dimensionless radius $\lambda$ and time $\tau$ :

$$
\lambda=\frac{r}{r_{o}} \quad \text { and } \quad \tau=\frac{c_{o} t}{r_{o}} .
$$

From the numerical solution for a cylindrical blast wave at the weak shock front, the size and average temperature of the hot (low-density) region near the origin, which may be identified as the initial kernel in spark ignition, are found at the appropriate transition time that thermal diffusion begins to dominate the mechanism of the kernel growth. The transition is expected to occur towards the end of an acoustic wave period. The relation between the Mach number and the dimensionless time is

$M \sim \frac{r_{o} / t}{c_{o}} \sim \frac{1}{\tau}$.

For an order-of-magnitude approximation, assume $\frac{1}{2}<M<1$ as a bound of an acoustic wave, which implies $1<\tau<2$. Then, $\tau=1.5$ may be a reasonable estimation for the transition time. The same value has already been used by $\mathrm{Lim}$ et al. [7, 8], however, without any dimensional justification. Plooster's numerical solution for the cylindrical blast wave [42] also shows that the blast wave diminishes to an approximate acoustic wave by this dimensionless time $\tau=1.5$, leaving behind a hot core near the center that expands no further in size. Considering $1 \mathrm{~mJ}$ of input energy over a 2-mm gap and normal temperature and pressure of air, from the above definition of $r_{o}$ and $\tau$,

$$
\begin{aligned}
r_{o} & =\left(\frac{E_{o}}{B \gamma P_{o}}\right)^{1 / 2} \\
& =\left[\frac{\left(1 \times 10^{-3} / 2 \times 10^{-3}\right)}{(3.94)(1.4)\left(1.013 \times 10^{5}\right)}\right]^{1 / 2} \\
& =9.5 \times 10^{-4} \mathrm{~m}
\end{aligned}
$$

and

$t=\frac{\tau r_{o}}{c_{o}}=\frac{(1.5)\left(9.5 \times 10^{-4}\right)}{350}=4 \mu \mathrm{s}$.

Therefore, $\tau=1.5$ corresponds to $4 \mu \mathrm{s}$, which is of the same order as the result from dimensional arguments above. The kernel radius at this time is on the order of $1 \mathrm{~mm}$, which is supported by schlieren photography. 
A kernel boundary in the blast wave is fixed at a radius $r_{f}$, where the density assumes $90 \%$ of the undisturbed value $\rho_{o}$ and the density gradient becomes small. This would approximate the boundary detected by schlieren photography. The total mass inside the kernel is found from an integration of the density profile in Ref. 42,

$m=\rho_{o} \int_{0}^{r_{f}} 2 \pi\left(\frac{\rho}{\rho_{o}}\right) r d d r$,

where $d$ denotes a spark gap size. The average temperature of this ideal gas kernel is

$T=\left(\frac{P V}{\gamma-1}\right) /\left(m C_{v}\right)=\frac{P V}{m \mathscr{R}}$,

where $\mathscr{R}$ is a gas constant. Because of the high temperature near the center of the blast up to this point in time, all the fuel in the kernel is assumed to be burned, increasing the size and temperature accordingly. The contribution of this chemical energy release is significant $[7,8,13,43]$. The initial kernel having the above properties is a result of the blast wave generated by the highpower input during the electrical breakdown of the gap.

\section{DIFFUSION MODEL}

Kernel growth after the blast wave is modeled as a spherical diffusion penetration (boundary layer) with an initial condition taken from the blast wave. A lean fuel-air mixture of the heavy gaseous fuel $A$ is considered ( $\mathrm{Le}_{A}=$ const $>1$ ) and the corresponding schematic of the flame structure is shown in Fig. 1. The assumptions are the burned gas temperature in zone $\mathrm{I}$ is uniform, the heating zone II is frozen in chemical reaction with the large activation energy assumption, which is true for most gaseous fuels, the fuel burns immediately after it enters the reaction zone, and the concentration of the fuel in the burned gas zone I is negligible as a result.

The integral formulations of the conservation of mass and the balance of thermal energy are, for the burned gas zone I,

$$
\frac{d}{d t} \int_{0}^{r_{f}} \rho 4 \pi r^{2} d r-\left(\dot{m}_{r_{f}} 4 \pi r_{f}^{2}+\dot{m}_{e}\right)=0 \text {, }
$$

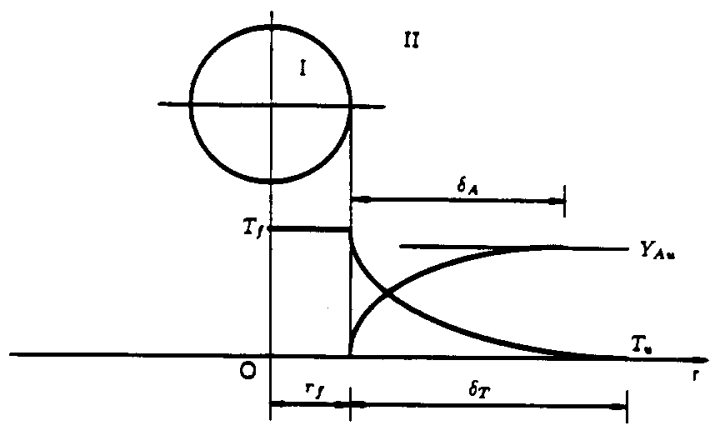

Fig. 1. Schematic of flame structure: profile of temperature and mass fraction of $A$.

$$
\begin{aligned}
\frac{d}{d t} \int_{0}^{r_{f}} \rho h 4 \pi r^{2} d r+q_{r_{f}} 4 \pi r_{f}^{2} \\
\quad-\left(\dot{m}_{r_{f}} 4 \pi r_{f}^{2}+\dot{m}_{e}\right) h_{r_{f}} \\
\quad-\dot{E}_{e}-Q_{A}\left(\dot{m}_{r_{f}}^{A} 4 \pi r_{f}^{2}+\dot{m}_{e}^{A}\right)=0,
\end{aligned}
$$

and for the heating zone II,

$$
\begin{aligned}
& \frac{d}{d t} \int_{r_{f}}^{r_{f}+\delta_{T}} \rho 4 \pi r^{2} d t+\dot{m}_{r_{f}} 4 \pi r_{f}^{2} \\
& -\dot{m}_{r_{f}+\delta_{T}} 4 \pi\left(r_{f}+\delta_{T}\right)^{2}=0, \\
& \frac{d}{d t} \int_{r_{f}}^{r_{f}+\delta_{T}} \rho h 4 \pi r^{2} d r-q_{r_{f}} 4 \pi r_{f}^{2} \\
& +\left(\dot{m}_{r_{f}} 4 \pi r_{f}^{2}+\dot{m}_{e}\right) h_{r_{f}} \\
& -\left(\dot{m}_{r_{f}+\delta_{T}} 4 \pi\left(r_{f}+\delta_{T}\right)^{2}+\dot{m}_{e}\right) h_{r_{f}+\delta_{T}}=0
\end{aligned}
$$

where $h$ is the enthalpy of the mixture, $r_{f}$ the flame kernel radius, $\delta_{T}$ the heating zone thickness (thermal penetration depth), $\delta_{A}$ the fuel penetra tion depth, $\dot{m}$ the mass flow rate of the mixture per unit area, $\dot{m}_{e}$ the entrainment mass flow rate due to the electrical discharge, $q_{r_{f}}$ the rate of heat conduction per unit area at $r=r_{f}$, and $\dot{E}$ the electrical input power. The discharge induced entrainment $\dot{m}_{e}$ is discussed in detail later. 
An ideal gas relation at constant pressure

$\rho T=$ const

is assumed. Also, the usual diffusion laws give

$\dot{m}_{r f}^{A}=\left.\rho D_{A} \frac{\partial Y_{A}}{\partial r}\right|_{r=r_{f}^{+}} \quad$ and

$q_{r_{f}}=-\left.k \frac{\partial T}{\partial r}\right|_{r=r_{f}}$.

The temperature and fuel concentration profiles in the heating zone II are assumed to be parabolic. The assumed profiles are

$$
T=\left\{\begin{array}{cc}
T_{f} \quad 0 \leq r \leq r_{f} \\
T_{u}+\left(T_{f}-T_{u}\right) \\
\times\left(\frac{\left(r_{f}+\delta_{T}\right)-r}{\delta_{T}}\right)^{2} \\
r_{f} \leq r \leq r_{f}+\delta_{T} \\
T_{u} \quad r_{f}+\delta_{r} \leq r
\end{array}\right.
$$

and

$$
Y_{A}=\left\{\begin{array}{cc}
0 \quad 0 \leq r \leq r_{f} \\
Y_{A_{u}}\left(1-\left(\frac{\left(r_{f}+\delta_{A}\right)-r}{\delta_{A}}\right)^{2}\right) \\
r_{f} \leq r \leq r_{f}+\delta_{A} \\
Y_{A_{u}} \quad r_{f}+\delta_{A} \leq r
\end{array}\right.
$$

The rate of fuel entering the burned gas zone I per unit area by molecular diffusion, $\dot{m}_{r_{f}}^{A}$, depends on both the geometry of the kernel as well as strongly on the burned gas temperature $T_{f}$ when the activation energy $\mathscr{E}$ is large $(\mathscr{E} \gg \Re T)$. From the experimental observations for kernel growth in Part I [1], the diffusive growth after the blast wave is conceived as having two different characteristics depending on the kernel radius. $\dot{m}_{r_{f}}^{A}\left(T_{f}\right)$ accounts for this difference in the present study. For a planar flame propagating with constant velocity, the dependence of the fuel burning rate per unit area, $\dot{m}_{x_{f}}^{A}$, on the constant flame temperature, $T_{f}$, [44] is

$\dot{m}_{x_{f}}^{A} \sim \alpha^{1 / 2} \exp \left(-\frac{\mathscr{E}}{2 \Re T_{f}}\right)$.

Introducing the fuel burning rate of the adiabatic flame to Eq. 25,

$$
\begin{aligned}
\dot{m}_{x_{f}}^{A}= & \rho_{u} U_{a} Y_{A_{u}}\left(\frac{(\alpha)_{T_{f}}}{(\alpha)_{T_{a}}}\right)^{1 / 2} \\
& \times \exp \left(-\frac{\mathscr{E}}{2 \Re}\left(\frac{1}{T_{f}}-\frac{1}{T_{a}}\right)\right) .
\end{aligned}
$$

Equation 26 can be used for a planar flame propagating with varying velocity, i.e., for varying flame temperature $T_{f}$, assuming quasi-steadiness.

For a spherical flame, there exists a steady critical flame $[15,31,32]$. This flame is unique to the spherical geometry and is expected to control the flame kernel growth. The spherical flame exhibits different characteristics than a planar flame. The dependence of the fuel burning rate per unit area, $\dot{m}_{r_{f}}{ }^{A}$, on the flame temperature, $T_{f}$, is derived from expressions for the steady critical flame. Using the profiles of fuel concentration (Eq. 9) as well as the expression for the critical radius (Eq. 18), the fuel burning rate for the nonsteady spherical flame with the quasisteady assumption becomes

$$
\begin{aligned}
\dot{m}_{r_{f}}^{A}= & \rho_{u} U_{a} Y_{A_{u}}\left(\frac{(\alpha)_{T_{f}}}{(\alpha)_{T_{a}}}\right) \\
& \times \exp \left(-\frac{\mathscr{E}}{2 \Re}\left(\frac{1}{T_{f}}-\frac{1}{T_{a}}\right)\right) .
\end{aligned}
$$

Equation 27 is valid when the kernel radius is small and of the order of the critical radius. This occurs when the kernel shows strong spherical flame characteristics with a smaller radius. On the other hand, Eq. 26 is valid when the kernel radius is much larger than the critical radius, or, when the kernel loses spherical flame characteristics and exhibits planar flame characteristics.

The expressions containing $\delta_{A}$ for each case 
are found from the definition of $\dot{m}_{r_{f}}{ }^{A}$ and the assumed profile $Y_{A}$ (Eq. 24):

$$
\begin{aligned}
\dot{m}_{r_{f}}^{A} & =\left.\rho D_{A} \frac{\partial Y_{A}}{\partial r}\right|_{r=r_{f}} \\
& =\left(\frac{1}{\mathrm{Le}_{A}}\right)\left(\frac{k}{C_{p}}\right)_{T_{f}}\left(\frac{2 Y_{A_{u}}}{\delta_{A}}\right),
\end{aligned}
$$

or,

$\delta_{A}=\left(\frac{1}{\mathrm{Le}_{A}}\right)\left(\frac{k}{C_{p}}\right)_{T_{f}}\left(\frac{2 Y_{A_{u}}}{\dot{m}_{r_{f}}^{A}}\right)$.

For a planar flame, assuming $T_{f}$ is close to $T_{a}$, from Eq. 26,

$$
\begin{aligned}
\delta_{A}= & 2 \frac{1}{\operatorname{Le}_{A}}\left(\frac{k}{C_{p}}\right)_{T_{a}} \frac{1}{\rho_{u} U_{a}} \\
& \times \exp \left(\frac{\mathscr{E}}{2 \Re}\left(\frac{1}{T_{f}}-\frac{1}{T_{a}}\right)\right)
\end{aligned}
$$

and for a spherical flame, from Eqs. 27 and 28,

$\delta_{A}=\left(\delta_{A}\right)_{\text {planar }}\left(\frac{T_{a}}{T_{f}}\right)$.

From Eqs. 28 and 29,

$$
\frac{d \delta_{A}}{d t}=C_{A} \delta_{A} \frac{d T_{f}}{d t},
$$

where

$$
C_{A}=\left\{\begin{array}{l}
-\frac{\mathscr{E}}{2 \Re T_{f}^{2}} \\
\text { for the planar flame, } \\
-\frac{1}{T_{f}}-\frac{\mathscr{E}}{2 \Re T_{f}^{2}} \\
\text { for the spherical flame. }
\end{array}\right.
$$

The term $\dot{m}_{e}$ is an additional inflow rate of unburned mixture entering the burned gas zone I around the electrodes while the spark is discharging. It is evidenced from the schlieren pictures of the experimental study and also in Refs. 7 and
43. This entrainment helps to explain the rapid kernel growth in the early stage of the diffusion process. Lim et al. [7, 8] suggest that the rate of entrainment, $\dot{m}_{e}$ be expressed as

$\dot{m}_{e} \sim \rho_{u} A_{e} u_{e}=C_{e} \rho_{u} A_{e} u_{e}=\rho_{u} C_{d} u_{e}$,

where $u_{e}$ is a representative entrainment velocity generated by the electrical power input and $C_{e}$ is a proportional constant. The entrainment area, $A_{e}$ is assumed to be constant as an approximation and $C_{d}$ is a proportional constant that contains the entrainment area. $u_{e}$ is derived from an assumption that some unknown fraction of the electrical power input $\dot{E}_{e}$ is largely converted to a kinetic energy flow of unburned gas into the kernel at a quasi-steady condition

$\dot{E}_{e} \approx\left(4 \pi r_{f}^{2}\right) \rho_{u} u_{e}^{3} \quad$ or $u_{e} \approx\left(\frac{\dot{E}_{e}}{4 \pi r_{f}^{2} \rho_{u}}\right)^{1 / 3}$

Equations 19-22 are rearranged and integrated. Eliminating $\dot{m}_{r_{f}}$ in Eqs. 19 and 20, and using Eqs. 23 and 24, the resulting differential equation for the burned gas zone $I$ is

$$
\begin{aligned}
\frac{r_{f}}{3}\left(\frac{\left(C_{p}\right)_{T_{f}}}{T_{f}}+\frac{d\left(C_{p}\right)_{T_{f}}}{d T_{f}}\right) \frac{d T_{f}}{d t} \\
=\frac{Q_{A} Y_{A_{u}}}{\rho_{b} T_{b}}\left(\frac{1}{\mathrm{Le}_{A}}\left(\frac{k}{C_{p}}\right)_{T_{f}} \frac{2}{\delta_{A}}+\frac{\dot{m}_{e}}{4 \pi r_{f}^{2}}\right) \\
\quad+\frac{\dot{E}_{e}}{4 \pi r_{f}^{2} \rho_{b} T_{b}}-\frac{(k)_{T_{f}}}{\rho_{b} T_{b}} \frac{2\left(T_{f}-T_{u}\right)}{\delta_{T}}
\end{aligned}
$$

Eliminating $\dot{m}_{r_{f}^{+} \delta_{T}}$ in Eqs. 21 and 22 and substituting Eq. 19 into the resulting equation gives

$$
\begin{aligned}
\int_{r_{f}}^{r_{f}+\delta} T \frac{\partial}{\partial t}\left(\rho C_{p} T\right) 4 \pi r^{2} d r \\
\quad-\int_{r_{f}}^{r_{f}+\delta_{T}} h_{u} \frac{\partial}{\partial t}(\rho) 4 \pi r^{2} d r \\
\quad-q_{r_{f}} 4 \pi r_{f}^{2}-(\rho)_{T_{f}} \frac{1}{3} \frac{r_{f}}{T_{f}} \frac{d T_{f}}{d t} \\
\times 4 \pi r_{f}^{2}\left(h_{T_{f}}-h_{u}\right)=0 .
\end{aligned}
$$


Substituting 23 and 24 , and a polynomial fit of $C_{p}$ in terms of temperature into Eq. 32 results in the differential equation for the heating zone II,

$$
C_{1} \frac{d T_{f}}{d t}+C_{2} \frac{d r_{f}}{d t}+C_{3} \frac{d \delta_{T}}{d t}=C_{4},
$$

where $C_{1}, C_{2}, C_{3}$, and $C_{4}$ are nonlinear coefficients. The derivation of Eq. 33 and the expressions for the coefficients $C_{1}$ through $C_{4}$ are given in the Appendix.

One additional equation is needed and obtained from the conservation of fuel and energy at $r=$ $r_{f}{ }^{+}$. In this way, an algebraic relation rather than a nonlinear differential equation is obtained, while retaining the physics of the problem at the most important point $\left(r=r_{f}^{+}\right)$. At $r=r_{f}^{+}$, there is no electrical discharge and chemical reactions are frozen with the large activation energy assumption. The conservation equations to be solved at $r=r_{f}{ }^{+}$for a convection-free thermodiffusion flame model with spherical coordinates, after substitution of the assumed profiles in Eqs. 23 and 24 , are

$$
\begin{aligned}
\frac{d T_{f}}{d t} & +\frac{2\left(T_{f}-T_{u}\right)}{\delta_{T}} \frac{d r_{f}}{d t} \\
& =\frac{2(k)_{T_{f}}\left(T_{f}-T_{u}\right)}{\left(\rho C_{p}\right)_{T_{f}} \delta_{T}}\left(\frac{1}{\delta_{T}}-\frac{2}{r_{f}}\right), \\
\frac{d r_{f}}{d t} & =\frac{\left(\rho D_{A}\right)_{T_{f}}}{(\rho)_{T_{f}}}\left(\frac{1}{\delta_{A}}-\frac{2}{r_{f}}\right) .
\end{aligned}
$$

Eqs. 34 and 35 are solved simultaneously with Eq. 31 to eliminate $d T_{f} / d t$ and $d r_{f} / d t$. The resulting algebraic relation is

$C_{5} \delta_{T}^{2}+C_{6} \delta_{T}+C_{7}=0$,

where $C_{5}, C_{6}$, and $C_{7}$ are nonlinear coefficients. Differentiating Eq. 36 with respect to time $t$ results in

$$
\frac{d \delta_{T}}{d t}=C_{8} \frac{d T_{f}}{d t}+C_{9} \frac{d r_{f}}{d t}+C_{10} \frac{d \delta_{A}}{d t}+C_{11},
$$

where $C_{8}, C_{9}, C_{10}$, and $C_{11}$ are nonlinear coef- ficients. The derivation of Eqs. 36 and 37 and the expressions for the coefficients $C_{5}$ through $C_{11}$ are given in the Appendix. Substituting Eqs. 30 and 37 into Eq. 33 results in

$$
\begin{aligned}
\left(C_{1}\right. & \left.+C_{3} C_{8}+C_{3} C_{10} C_{A} \delta_{A}\right) \frac{d T_{f}}{d t} \\
& +\left(C_{2}+C_{3} C_{9}\right) \frac{d r_{f}}{d t}=C_{4}-C_{3} C_{11} .
\end{aligned}
$$

There are two ordinary differential Eqs. 31 and 38 to be solved for the unknowns $\left(T_{f}, r_{f}\right)$ with the algebraic relations (Eqs. 28 or 29 , and 36 ). These equations are numerically solved with the initial conditions specified from the blast wave solution in order to simulate the spark kernel development. The time-dependent spark power $\dot{E}_{e}$ can either be assumed or taken from measurements.

The numerical integration starts with the fuel burning rate relation (Eq. 27) for the spherical flame. When either the kernel temperature starts increasing with the radius larger than the critical radius or when the kernel radius reaches $1.5 \times r_{c}$, the fuel burning rate relation (Eq. 26) for the planar flame is used in the integration. Because the temperature of a steady spherical flame $T_{b}$ is lower than the planar adiabatic flame temperature $T_{a}$ when $\mathrm{Le}_{A}>1$ (see Eq. 5), the increasing kernel temperature when the radius is larger than the critical value implies that the effect of the steady spherical flame on the kernel growth is diminishing. The criterion for the transition radius, $1.5 \times r_{c}$, is assumed from the experimental observations in Ref. 9 and Part I [1]: the flame speed starts increasing around the radius $1.5 \times r_{c}$. This implies that the kernel radius of $1.5 \times r_{c}$ is large enough to neglect the effect of the steady spherical flame on the kernel growth.

The properties of air are used for both the unburned mixture and burned gas. The enthalpy and the constant pressure specific heat, $C_{p}$, are computed from those of $\mathrm{N}_{2}$ and $\mathrm{O}_{2}$ in the JANAF tables, and the temperature dependence of $C_{p}$ as a fourth-order polynomial fit is used for integration of the conservation equations. The thermal conductivity of air is taken from Yos [45].

For propane-air mixtures, the activation en- 


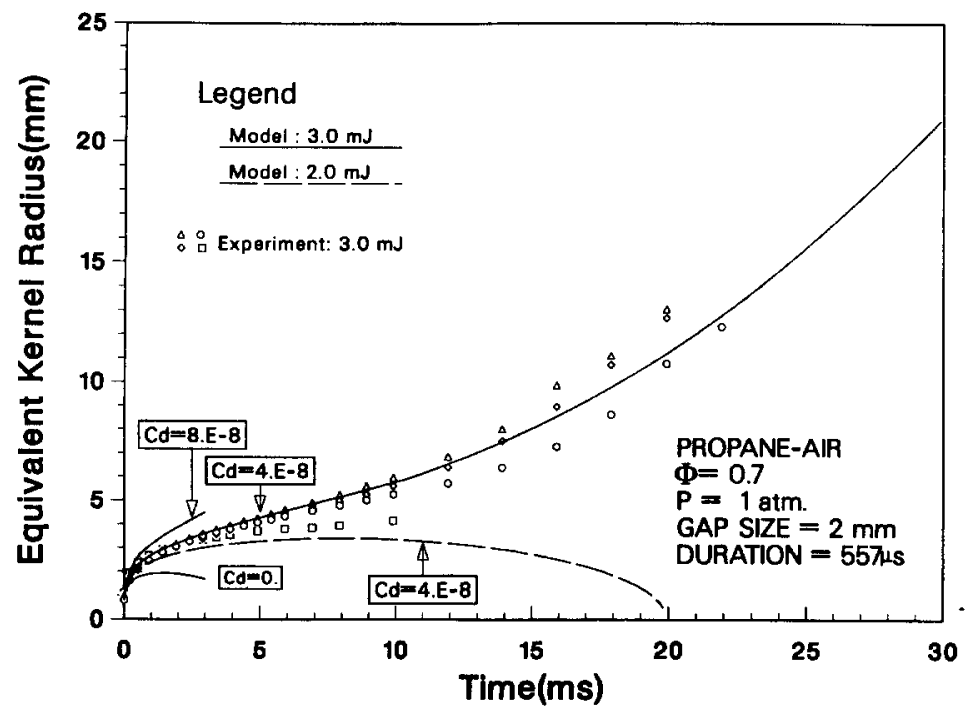

Fig. 2. Comparison of predicted and measured kernel radii in a propane-air mixture ( $\phi=0.7, d=2 \mathrm{~mm}$, and $t_{d}=557 \mu \mathrm{s}$ ).

ergy $\mathscr{E}=37,700 \mathrm{cal} / \mathrm{mole}$ [46] is used and the adiabatic laminar burning velocity $U_{a}$ is taken from the correlation of Lavoie [47]. The adiabatic laminar burning velocity at $\phi=0.6$ is estimated from the polynomial fit of the data at $\phi=0.9$, $0.8,0.7$, and 0.53. $\phi \approx 0.53$ is the lean limit [48] where the flame speed is assumed to be zero.

\section{DISCUSSION OF MODEL PREDICTIONS}

The model developed in Sec. 4 is run to simulate the experimentally observed kernel growth in Part I [1]. The actual available spark power for ignition is estimated from the measured total spark power using Eq. 4 in Sec.4 of Part I [1].

The model developed in Sec. 4 has a constant $C_{d}$ to be calibrated. The discharged induced inflow term, which includes the constant $C_{d}$, affects both the kernel temperature and the kernel growth. Larger values of $C_{d}$ result in higher kernel temperatures and faster kernel growth rates. The effect is pronounced during the early stage of the kernel development. A calibration is made with the measured kernel radius, breakdown energy, and spark energy curve for the case of $\phi=0.7$ and $d=2 \mathrm{~mm}$ presented in Fig. 2 of the experimental study of Part I [1]. The constant
$C_{d}$ is adjusted until the predicted kernel radius matches the experimentally determined curve in the early stage of kernel development. As shown in Fig. 2, a reasonable fit in the early stage including the starting point from the blast wave solution is achieved with the constant $C_{d}=4 \times$ $10^{-8} \mathrm{~m}^{2}$, which is used in the remainder of the study. Lim and colleagues $[7,8]$ use $C_{d}=8 \times$ $10^{-8} \mathrm{~m}^{2}$ in their model. The difference between the two models is a result of the fact that the higher kernel temperature resulting from the discharge-induced inflow causes a higher fuel burning rate per unit area by molecular diffusion in the present model (Eqs. 26 or 27); however, it does not have any effect on the fuel burning rate per unit area in the model of $\mathrm{Lim}$ et al. The previous study also utilizes a different electrode geometry.

In addition to model calibration, Fig. 2 shows the prediction of the flame kernel growth up to 30 $\mathrm{ms}$. The quenching effect in this case is small due to a larger gap size $(2 \mathrm{~mm})$, as shown in Sec. 4 of Part I [1] and the discharge duration $(557 \mu \mathrm{s})$ is small enough to make the effect of time losses (i.e., $\eta_{d}(t)$ in Eq. 4 of Part I [1]) insignificant on the ignition process. The model predicts the kernel growth reasonably well, including the slower 


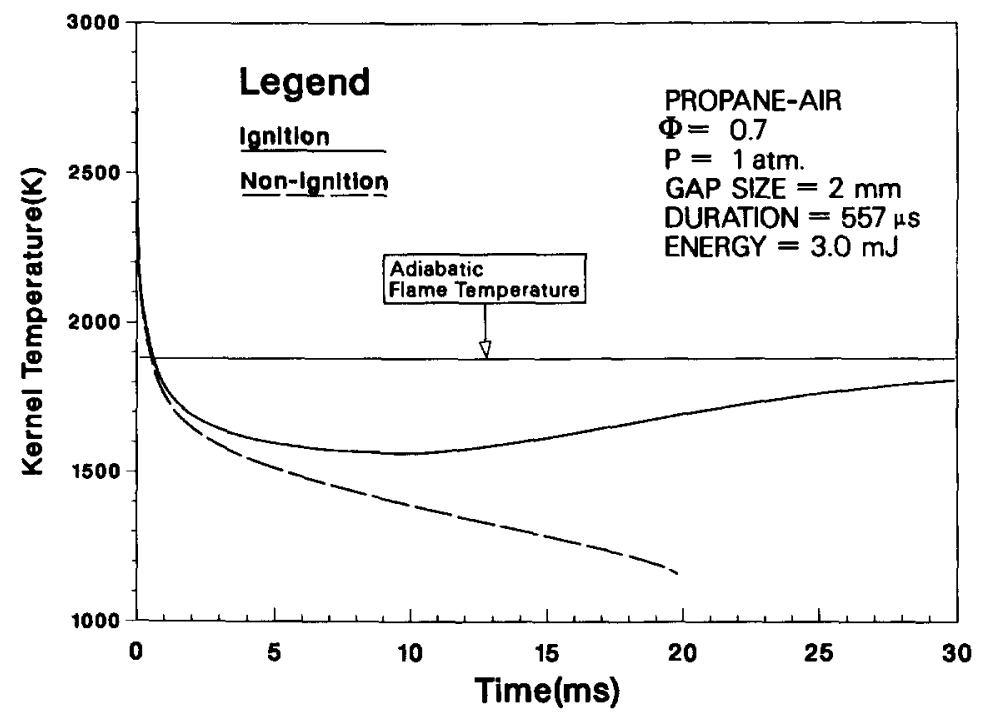

Fig. 3. Predicted kernel temperature in a propane-air mixture $(\phi=0.7, d=2 \mathrm{~mm}$, and $t_{d}=557 \mu$ s (Fig. 2)).

kernel growth around the critical radius and the transition from the slower flame speed to the planar adiabatic flame speed.

In Fig. 2, the model also predicts nonignition kernel growth with a measured spark energy curve that has a near-zero ignition probability. The spark energy level in this prediction is substantially lower than the minimum ignition energy at this condition. The model predicts a kernel growth in the early stage that is similar to that for the ignition case with the minimum ignition energy. The insufficient spark energy, however, fails to drive the kernel to a radius larger than the critical radius and the kernel is eventually extinguished. This is similar to experimental observations for the nonignition case with the minimum ignition energy in Part I [1]. Because the present model is calibrated to predict the flame kernel growth of the ignition case at conditions near the minimum ignition energy, it cannot simultaneously predict both ignition and nonignition phenomena, as observed in the experimental study of Part I [1] with the near minimum ignition energy. The occurrence of ignition or nonignition at conditions near the minimum ignition energy has a statistical variation resulting from complex nonlinear processes. The spark energy that results in ignition at conditions near the minimum ignition energy may give nonignition in the next trial in actual cases. The prediction of this kind of statistical variation in spark ignition is beyond the ability of this simplified thermodiffusion ignition model. The present model, however, does predict nonignition phenomena when using the measured spark energy, which experimentally has a zero ignition probability.

Figure 3 shows typical temporal variations of the average kernel temperature from the model for the ignition and nonignition conditions of Fig. 2 . For the ignition case near the minimum ignition energy, the kernel temperature decreases immediately after the blast wave, falls below the adiabatic flame temperature, and later begins to increase towards the adiabatic flame temperature. The temperature is much lower than the adiabatic temperature when the kernel radius is close to the critical radius, which appears to result in a slower kernel growth rate. When the kernel radius is small, the temperature decreases from the initial high temperature caused by the breakdown event because the rate of conduction heat loss to the unburned fresh mixture is larger than the rate of combustion energy release plus the input of electrical discharge energy. The kernel temperature 


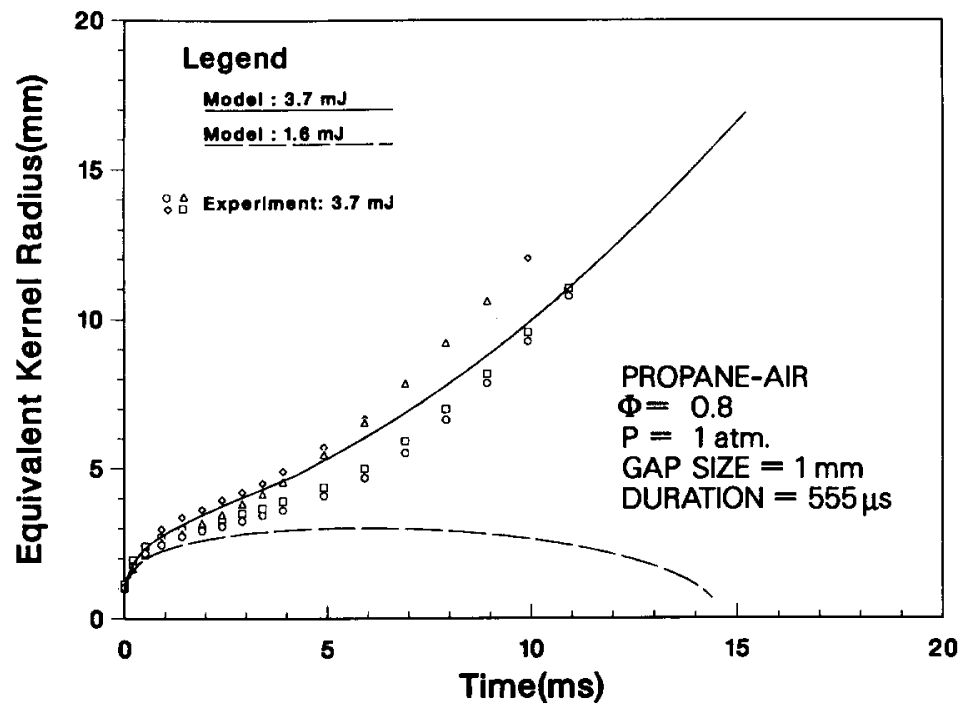

Fig. 4. Comparison of predicted and measured kernel radii in a propane-air mixture $\left(\phi=0.8, d=1 \mathrm{~mm}\right.$, and $\left.t_{d}=555 \mu \mathrm{s}\right)$.

starts to increase when the kernel grows large enough to cause the rate of combustion energy release to exceed the rate of conduction heat loss. For this ignition case, the trends of the kernel temperature variations agree with those presented in Ref. 15. For the nonignition case, the kernel temperature decreases monotonically toward the ambient temperature. This is because the kernel never reaches the critical radius as a result of insufficient spark energy. The above predictions of kernel temperature confirm that for ignition to occur, the electric discharge must be sufficient to drive the kernel beyond the critical radius where the rate of combustion energy release starts exceeding the rate of conduction loss.

The model is run to test the predictions at other conditions. Figure 4 presents model predictions corresponding to the experimental case of $\phi=$ $0.8, d=1 \mathrm{~mm}$, and $t_{d}=550 \mu \mathrm{s}$ (Fig. 4 of Part I [1]) with $\eta_{g}=0.8$. Figure 5 presents similar predictions for the experimental case of $\phi=0.6$, $d=2 \mathrm{~mm}$, and $t_{d}=3.4 \mathrm{~ms}$ (Fig. 5 of Part I [1]) with $\eta_{g}=0.78$. The spark energy curves used for prediction of nonignition are the measured ones with near $0 \%$ ignition probability for each case. The discharge duration is very long for the $\phi=0.6$ case and the spark energy contributing to ignition is about $10.1 \mathrm{~mJ}$, or $27.2 \%$ from a positive column energy $E_{p}$ of $37.1 \mathrm{~mJ}$. This represents an overall efficiency of only $16.6 \%$ from the measured energy level of $61.0 \mathrm{~mJ}$ and compares favorably with the efficiency data of Teets and Sell [49]. From these results, it appears that the model predicts the kernel growth for both ignition and nonignition cases reasonably well for various conditions. Furthermore, the model predicts the kernel growth during the discharge period very well, as in other case (see Ko [9] for more results) and supports the adequacy of the constant $C_{d}$ calibrated in Fig. 2.

The burning rate of mixtures in the model is based on simple overall temperature-dependent Arrhenius reaction kinetics. It is derived from characteristics of either the critical spherical flame or the steady planar flame, depending on the kernel radius. The good predictions of the model for kernel growth, despite many simplifications, confirm that the kernel growth is controlled by the physics of the critical spherical flame when the kernel radius is less than or close to the critical radius [15]. The existence of the critical spherical flame, therefore, characterizes the flame kernel growth during the discharge period in spark ignition, as evidenced in both the experi- 


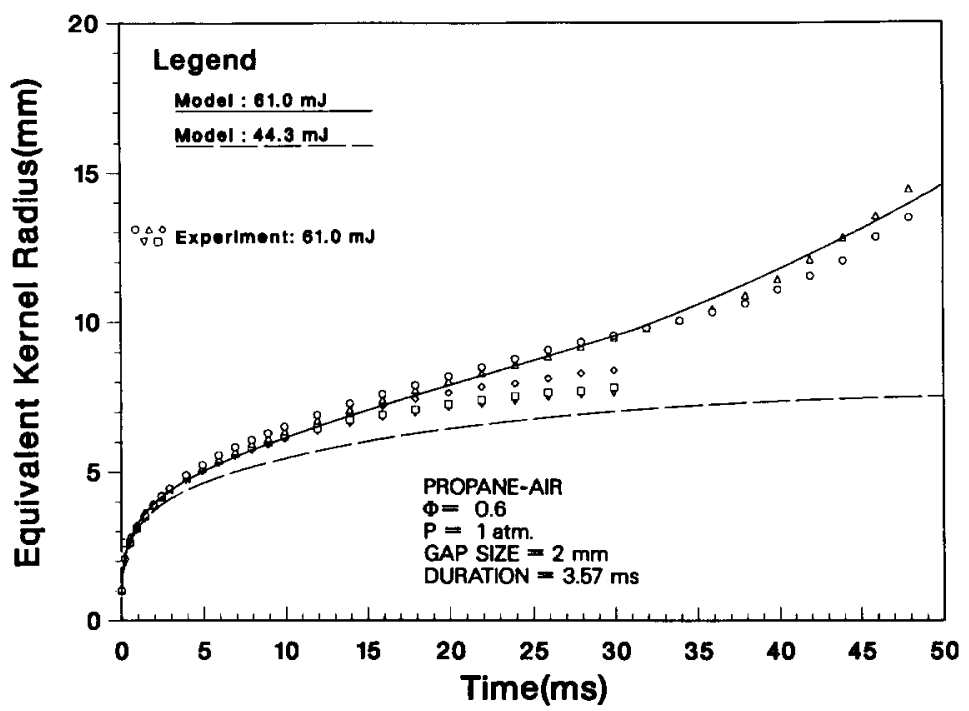

Fig. 5. Comparison of predicted and measured kernel radii in a propane-air mixture ( $\phi=0.6, d=2 \mathrm{~mm}$, and $t_{d}=3.57 \mathrm{~ms}$ ).

mental study of Part I [1] and the present modeling study.

\section{SUMMARY AND CONCLUSIONS}

Based on experimental observations and the characteristics of both spherical and planar flames, a model is developed to simulate the flame kernel growth. The model predicts ignition and nonignition growth of the flame kernel reasonably well with the measured spark power input, including the slower kernel growth around the critical radius and the transition from the slower flame speed regime to the planar adiabatic flame speed with a single constant adjusted at one condition. Kernel growth in the present model is described as a two-step process. The model initially involves a blast wave over a negligible short time followed by diffusive growth with an electrical input power. The diffusive growth is formulated by an integral approach involving temperature dependent overall reaction kinetics and a thermodiffusion model. Losses due to quenching, discharge duration, and the electrode fall energy are taken into consideration.

The model uses the characteristics of both the critical spherical flame when the kernel radius is small and the steady planar flame when the kernel radius is large. The good predictions of kernel growth by the model, therefore, confirm that the early flame kernel growth in spark ignition is controlled by the physics of the critical spherical flame.

The dimensionless critical radius $R_{c}$ found analytically by Champion et al. [15] is obtained from simple dimensional arguments. The characteristic radius $r_{o}$ of the blast wave due to an instantaneous energy release is also obtained by dimensional arguments.

\section{REFERENCES}

1. Ko, Y., Anderson, R. W., and Arpaci, V. S., Combust. Flame 83:75-87 (1991).

2. Lewis, B., and von Elbe, G., J. Chem. Phys. 15:803-808 (1947).

3. Ballal, D. R., and Lefebvre, A. H., Proc. R. Soc. Lond. A 357:163-181 (1977).

4. Ballal, D. R., and Lefebvre, A. H., Eighteenth Symposium (International) on Combustion, The Combustion Institute, Pittsburgh, 1981, pp. 1737-1746.

5. Swett, C. C., Jr., NACA T.R. 1287:799-816 (1956).

6. Fenn, J. B., Ind. Eng. Chem. 43:2865-2869 (1951).

7. Lim, M. T., Ph.D. thesis, The University of Michigan, Ann Arbor, 1985 
8. Lim, M. T., Anderson, R. W., and Arpaci, V. S., Combust. Flame 69:303-316 (1987).

9. Ko, Y., Ph.D. thesis, The University of Michigan, Ann Arbor, 1989.

10. de Soete, G. G., Thirteenth Symposium (International) on Combustion, The Combustion Institute, Pittsburgh, 1971, pp. 735-743.

11. Adelman, H. G., Eighteenth Symposium (International) on Combustion, The Combustion Institute, Pittsburgh, 1981, pp. 1333-1342.

12. Maly, R., Eighteenth Symposium on Combustion, The Combustion Institute, Pittsburgh, 1981, pp. 1747-1754.

13. Akindele, O. O., Bradley, D., Mak, P. W., and McMahon, M., Combust. Flame 47:129-155 (1982).

14. Bradley, D., and Lung, F. K-K., Combust. Flame 69:71-93 (1987).

15. Champion, B., Deshaies, B., Joulin, G., and Kinoshita, K., Combust. Flame 65:319-337 (1986).

16. Joulin, G., Combust. Sci. Technol. 43:99-113 (1985).

17. Sloane, T. M., Combust. Sci. Technol. 42:131-144 (1985).

18. Overley, J. R., and Overholser, K. A., Combust. Flame 31:69-83 (1978).

19. Dixon-Lewis, G. and Shepherd, I. G., Fifteenth Symposium (International) on Combustion, The Combustion Institute, Pittsburgh, 1975, pp. 1483-1491.

20. Tromans, P. S., and Furzeland, R. M., Twenty-First Symposium (International) on Combustion, The Combustion Institute, Pittsburgh, 1986, pp. 1891-1897.

21. Sher, E., and Refael, S., Nineteenth Symposium (International) on Combustion, The Combustion Institute, Pittsburgh, 1982, pp. 251-257.

22. Refael, S., and Sher, E., Combust. Flame 59:17-30 (1985).

23. Sher, E., and Keck, J. C., Combust. Flame 66:17-25 (1986).

24. Kailasanath, K., Oran, E. S., and Boris, J. P., Combust. Flame 47:173-190 (1982).

25. Oran, E. S., and Boris, J. P., Seventh International Colloquium on Gas Dynamics of Explosions and Reactive Systems, Göttingen, 1979, pp. 154-171.

26. Rajan, S., Central States Section, The Combustion Institute, April, 1979.

27. Lutz, A. E., Kee, R. J., and Dwyer, H. A., Western States Section, The Combustion Institute, Joint Technical Meeting, San Antonio, April, 1985.

28. Maly, R., and Vogel, M., Seventeenth Symposium on Combustion, The Combustion Institute, Pittsburgh, 1979, pp. 821-831.

29. Albrecht, H., Bloss, W. H., Herden, W., Maly, R., Saggau, B., and Wagner, E., SAE Paper 770853 (1977).

30. Merzhanov, A. G., and Averson, A. E., Combust. Flame 16:89-124 (1971).

31. Deshaies, B., and Joulin, G., Combust. Sci. Technol. 37:99-116 (1984).

32. Zeldovich, Ya. B., Barenblatt, G. I., Librovich, B. V., and Makhviladze, G. M., The Mathematical Theory of Combustion and Explosions, Nauka, Moscow, 1980.

33. Lintin, D. R., and Wooding, E. R., Br. J. Appl. Phys. 10:159-166 (1959).

34. Arnold, J. S., and Sherburne, R. K., Fourth Symposium on Combustion, Williams \& Wilkins, Baltimore, 1953, pp. 139-143.

35. Olsen, H. L., Gayhart, E. L., and Edmonson, R. B., Fourth Symposium on Combustion, Williams \& Wilkins, Baltimore, 1953, pp. 144-148.

36. Bush, W. B., and Fendell, F. E., Combust. Sci. Technol. 1:421-428 (1970).

37. Hirschfelder, J. O., Curtiss, C. F., and Bird, R. B., Molecular Theory of Gases and Liquids, Wiley, New York, 1954.

38. Baritaud, T. A., SAE Paper 872152 (1987).

39. Taylor, G. I., Proc. R. Soc. Lond. A 201:159-174 (1950).

40. Sedov, L. I., Similarity and Dimensional Methods in Mechanics (translation; M. Holt, Ed.), Academic, New York, 1957.

41. Jones, D. L., Phys. Fluids 4:1183-1184 (1961) [see also Phys. Fluids 5:637 (1962) for correction].

42. Plooster, M. N., Phys. Fluids 13:2665-2675 (1970).

43. Anderson, R. W., and Lim, M. T., Eighth International Conference on Gas Discharges and Their Applications, Pergamon, London, 1985, pp. 511-514.

44. Glassman, I., Combustion, Academic, New York, 1977.

45. Yos, J. M., RAD-TM-63-7, March 1963.

46. Ferguson, C. R., and Keck, J. C., Combust. Flame 28:197-205 (1977).

47. Lavoie, G. A., SAE Paper 780229 (1978).

48. Lewis, B. L., and von Elbe, G., Combustion, Flames, and Explosions of Gases, Academic, New York, 1961.

49. Teets, R. E., and Sell, J. A., SAE Paper 880204 (1988).

Received 22 August 1989; revised 13 March 1990

\section{APPENDIX}

\section{Expressions of $C_{1}$ to $C_{4}$}

Equation 32 in Sec. 4 is integrated as follows:

$$
\begin{aligned}
& \int_{r_{f}^{+}}^{r_{f}^{+} \delta_{T}} \frac{\partial}{\partial t}\left(\rho C_{p} T\right) 4 \pi r^{2} d r \\
& \quad=4 \pi \rho_{b} T_{b} \int_{r_{f}^{+}}^{r_{f}^{+} \delta_{T}} \frac{\partial}{\partial t}\left(C_{p}\right) r^{2} d r \\
& \quad=4 \pi \rho_{b} T_{b} \int_{r_{f}^{+}}^{r_{f}^{+}+\delta_{T}} \frac{\partial}{\partial T}\left(C_{p}\right) \frac{\partial T}{\partial t} r^{2} d r
\end{aligned}
$$


is first integrated with $C_{p}$ as a fourth-order polynomial fit in terms of temperature and a temperature distribution in the region (Eq. 23). $C_{p}$ in terms of temperature $T^{*}=T-T_{r}$ is

$$
\begin{gathered}
C_{p}=B_{0}^{*}+B_{1}^{*} T^{*}+B_{2}^{*} T^{*^{2}} \\
+B_{3}^{*} T^{* 3}+B_{4}^{*} T^{* 4}
\end{gathered}
$$

and $\partial C_{p} / \partial T$ is expressed as

$$
\frac{\partial C_{p}}{\partial T}=B_{1}+B_{2} T^{*}+B_{3} T^{* 2}+B_{4} T^{* 3}
$$

where $B_{1}=B_{1}^{*}, B_{2}=2 B_{2}^{*}, B_{3}=3 B_{3}^{*}$ and $B_{4}$ $=4 B_{4}^{*}$, and $T_{r}$ is the reference temperature for the polynomial fit. The temperature distribution in the region (Eq. 23) is

$$
T=T_{u}+\left(T_{f}-T_{u}\right)\left(\frac{r_{f}+\delta_{T}-r}{\delta_{T}}\right)^{2}
$$

and gives

$$
\begin{aligned}
\frac{\partial T}{\partial t}= & {\left[\frac{d T_{f}}{d t}-\frac{2\left(T_{f}-T_{u}\right)}{\delta_{T}} \frac{d \delta_{T}}{d t}\right] } \\
& \times\left(\frac{r_{f}+\delta_{T}-r}{\delta_{T}}\right)^{2} \\
& +\left[\frac{2\left(T_{f}-T_{u}\right)}{\delta_{T}} \frac{d}{d t}\left(r_{f}+\delta_{T}\right)\right] \\
& \times\left(\frac{r_{f}+\delta_{T}-r}{\delta_{T}}\right) .
\end{aligned}
$$

Equation A1 becomes, after substituting Eqs. A2 and $\mathrm{A} 4$ into Eq. A1 and integrating,

$$
\begin{gathered}
4 \pi \rho_{b} T_{b}\left[N_{1} \frac{d T_{f}}{d t}+N_{2} \frac{2\left(T_{f}-T_{u}\right)}{\delta_{T}} \frac{d r_{f}}{d t}\right. \\
\left.+\left(N_{2}-N_{1}\right) \frac{2\left(T_{f}-T_{u}\right)}{\delta_{T}} \frac{d \delta_{T}}{d t}\right]
\end{gathered}
$$

where

$$
\begin{aligned}
N_{1}= & B_{5} m_{2} \delta_{T}+B_{6}\left(T_{f}-T_{u}\right) m_{4} \delta_{T} \\
& +B_{7}\left(T_{f}-T_{u}\right)^{2} m_{6} \delta_{T} \\
& +B_{8}\left(T_{f}-T_{u}\right)^{3} m_{8} \delta_{T},
\end{aligned}
$$

$$
\begin{aligned}
N_{2}= & B_{5} m_{1} \delta_{T}+B_{6}\left(T_{f}-T_{u}\right) m_{3} \delta_{T} \\
& +B_{7}\left(T_{f}-T_{u}\right)^{2} m_{5} \delta_{T} \\
& +B_{8}\left(T_{f}-T_{u}\right)^{3} m_{7} \delta_{T}, \\
B_{5}= & B_{1}+B_{2}\left(T_{u}-T_{r}\right)+B_{3}\left(T_{u}-T_{r}\right)^{2} \\
& +B_{4}\left(T_{u}-T_{r}\right)^{3}, \quad B_{8}=B_{4}, \\
B_{6}= & B_{2}+2 B_{3}\left(T_{u}-T_{r}\right)+3 B_{4}\left(T_{u}-T_{r}\right)^{2}, \\
B_{7}= & B_{3}+3 B_{4}\left(T_{u}-T_{r}\right),
\end{aligned}
$$$$
m_{1}=\frac{1}{2} r_{f}^{2}+\frac{1}{3} r_{f} \delta_{T}+\frac{1}{12} \delta_{T}^{2} \text {, }
$$$$
m_{2}=\frac{1}{3} r_{f}^{2}+\frac{1}{6} r_{f} \delta_{T}+\frac{1}{30} \delta_{T}^{2},
$$$$
m_{3}=\frac{1}{4} r_{f}^{2}+\frac{1}{10} r_{f} \delta_{T}+\frac{1}{60} \delta_{T}^{2},
$$$$
m_{4}=\frac{1}{5} r_{f}^{2}+\frac{1}{15} r_{f} \delta_{T}+\frac{1}{105} \delta_{T}^{2},
$$$$
m_{5}=\frac{1}{6} r_{f}^{2}+\frac{1}{21} r_{f} \delta_{T}+\frac{1}{168} \delta_{T}^{2},
$$$$
m_{6}=\frac{1}{7} r_{f}^{2}+\frac{1}{28} r_{f} \delta_{T}+\frac{1}{252} \delta_{T}^{2},
$$$$
m_{7}=\frac{1}{8} r_{f}^{2}+\frac{1}{36} r_{f} \delta_{T}+\frac{1}{360} \delta_{T}^{2},
$$$$
m_{8}=\frac{1}{9} r_{f}^{2}+\frac{1}{45} r_{f} \delta_{T}+\frac{1}{495} \delta_{T}^{2},
$$$$
\int_{r_{f}^{+}}^{r^{+}+\delta_{T}} \frac{\partial}{\partial t}(\rho) 4 \pi r^{2} d r
$$$$
=4 \pi \rho_{b} T_{b} \int_{r f^{+}}^{r_{f}+\delta T} \frac{\partial}{\partial t}\left(\frac{1}{T}\right) r^{2} d r
$$$$
=4 \pi \rho_{b} T_{b} \int_{r f^{+}}^{r f^{+} \delta} T\left(-\frac{1}{T^{2}}\right) \frac{\partial T}{\partial t} r^{2} d r
$$

is also integrated with the temperature distribution. Inserting Eqs. A3 and A4 into Eq. A6 and 
integrating Eq. A6 results in

$$
\begin{aligned}
\mathrm{Eq} \cdot \mathrm{A6}= & 4 \pi \rho_{b} T_{b}\left[A_{1} \frac{d T_{f}}{d t}\right. \\
& \left.+A_{2} \frac{d T_{f}}{d t}+A_{3} \frac{d \delta_{T}}{d t}\right],
\end{aligned}
$$

where

$$
\begin{aligned}
A_{1}= & \frac{\delta_{T}^{3}}{2\left(T_{f}-T_{u}\right)^{2}}\left[-3+\left(\frac{r_{f}}{\delta_{T}}\right)^{2}\left(1-\frac{T_{u}}{T_{f}}\right)\right. \\
& -2\left(1+\frac{r_{f}}{\delta_{T}}\right) \ln \left(\frac{T_{u}}{T_{f}}\right) \\
& -\left(\left(1+r_{f}+\delta_{T}\right)^{2}\left(\frac{T_{f}}{T_{u}}-1\right)-3\right) \\
& \times \frac{1}{\sqrt{\left(\frac{T_{f}}{T_{u}}-1\right)}} \tan ^{-1} \sqrt{\left(\frac{T_{f}}{T_{u}}-1\right)} \\
A_{2}= & \frac{\delta_{T}^{2}}{T_{u}}\left[\frac{1}{\left(\frac{T_{f}}{T_{u}}\right)-1} \ln \left(\frac{T_{u}}{T_{f}}\right)+2\left(1+\frac{r_{f}}{\delta_{T}}\right)\right. \\
\left.A_{3}\right) & -\frac{2\left(T_{f}-T_{u}\right)}{\delta_{T}} A_{1}+A_{2} \cdot \\
& \times \frac{1}{\sqrt{\left(\frac{T_{f}}{T_{u}}-1\right)}} \tan { }^{-1} \sqrt{\left(\frac{T_{f}}{T_{u}}-1\right)} \\
& \left.-\left(1+\frac{T_{u}}{T_{f}}\right)\left(\frac{r_{f}}{\delta_{T}}\right)\right], \\
& \\
& -(1)
\end{aligned}
$$

From the definition of ${q_{r f}}^{+}$and the temperature distribution (Eq. A3),

$q_{r_{f}}^{+}=-\left.(k)_{T_{f}} \frac{\partial T}{\partial r}\right|_{r=r_{f}}=-\frac{2(k) T_{f}\left(T_{f}-T_{u}\right)}{\delta_{T}}$.

Inserting Eqs. A5, A7, and A8 into Eq. 32

results in

$C_{1} \frac{d T_{f}}{d t}+C_{2} \frac{d r_{f}}{d t}+C_{3} \frac{d \delta_{T}}{d t}=C_{4}$,

where

$C_{1}=N_{1}-A_{1} C_{p_{u}} T_{u}$

$$
\begin{gathered}
-\frac{1}{3} \frac{r_{f}^{3}}{T_{f}^{2}}\left(\left(C_{p}\right)_{T_{f}} T_{f}-C_{p_{u}} T_{u}\right), \\
C_{4}=\frac{2(k)_{T_{f}}\left(T_{f}-T_{u}\right)}{\rho_{b} T_{b} \delta_{T}} r_{f}^{2}, \\
C_{2}=N_{2} \frac{2\left(T_{f}-T_{u}\right)}{\delta_{T}}-A_{2} C_{p_{u}} T_{u}, \\
C_{3}=\left(N_{2}-N_{1}\right) \frac{2\left(T_{f}-T_{u}\right)}{\delta_{T}}-C_{p_{u}} T_{u} A_{3} .
\end{gathered}
$$

\section{Expressions of $C_{5}$ to $C_{11}$}

Solving Eqs. 34, 35, and 31 simultaneously to eliminate $d T_{f} / d t$ and $d r_{f} / d t$ results in

$C_{5} \delta_{T}^{2}+C_{6} \delta_{T}+C_{7}=0$,

where

$$
\begin{aligned}
C_{5}= & C_{51}+C_{52}+C_{53}, \\
C_{51}= & \frac{Q_{A} Y_{A_{u}}}{\rho_{b} T_{b} \mathrm{Le}_{A}} \frac{2}{\delta_{A}}\left(\frac{k}{C_{p}}\right)_{T_{f}}, \\
C_{52}= & \frac{\dot{E}_{e}}{4 \pi \rho_{b} T_{b} r_{f}^{2}}, C_{53}=\frac{Q_{A} Y_{A_{u}}}{4 \pi \rho_{b} T_{b}} \frac{\dot{m}_{e}}{r_{f}^{2}}, \\
C_{7}= & -\frac{2 r_{f}}{3}\left(\frac{\left(C_{p}\right)_{T_{f}}}{T_{f}}\right. \\
& \left.\left.+\frac{d\left(C_{p}\right)_{T_{f}}}{d T_{f}}\right)_{\left(T_{f}\right.}-T_{u}\right)\left(\frac{k}{\rho C_{p}}\right)_{T_{f}}, \\
\dot{m}_{e}= & \rho_{u} C_{d}\left(\frac{\dot{E}_{e}}{4 \pi r_{f}^{2} \rho_{u}}\right)^{1 / 3},
\end{aligned}
$$




$$
\begin{aligned}
C_{6}= & -C_{7}\left(\frac{1}{\operatorname{Le}_{A}} \frac{1}{\delta_{A}}+\left(1-\frac{2}{\mathrm{Le}_{A}}\right) \frac{2}{r_{f}}\right) \\
& -\frac{2(k)_{T_{f}}\left(T_{f}-T_{u}\right)}{\rho_{b} T_{b}} .
\end{aligned}
$$

From Eq. 36,

$$
\begin{gathered}
\delta_{T}=\frac{-C_{6}+\sqrt{C_{6}^{2}-4 C_{5} C_{7}}}{2 C_{5}} \text { and } \\
\frac{d \delta_{T}}{d t}=\frac{-\delta_{T}^{2} \frac{d C_{5}}{d t}-\delta_{T} \frac{d C_{6}}{d t}-\frac{d C_{7}}{d t}}{2 C_{5} \delta_{T}+C_{6}}
\end{gathered}
$$

$$
\begin{aligned}
C_{6 r}= & -C_{7 r}\left(\frac{1}{\mathrm{Le}_{A}} \frac{1}{\delta_{A}}+\left(1-\frac{1}{\mathrm{Le}_{A}}\right) \frac{2}{r_{f}}\right) \\
& +C_{7}\left(1-\frac{1}{\mathrm{Le}_{A}}\right) \frac{2}{r_{f}^{2}},
\end{aligned}
$$

$$
\begin{aligned}
& C_{6 A}=C_{7} \frac{1}{\operatorname{Le}_{A}} \frac{1}{\delta_{A}^{2}}, \quad C_{7 r}=C_{7} \frac{1}{r_{f}}, \\
& C_{7 T}=C_{7}\left[\frac{1}{T_{f}-T_{u}}+\frac{1}{(k)_{T_{f}}} \frac{d(k)_{T_{f}}}{d T_{f}}\right. \\
& -\frac{1}{\left(C_{p}\right)_{T_{f}}} \frac{d\left(C_{p}\right)_{T_{f}}}{d T_{f}}+\frac{1}{T_{f}}
\end{aligned}
$$

From $C_{5}, C_{6}$, and $C_{7}$,

$$
\frac{d C_{5}}{d t}=C_{5 T} \frac{d T_{f}}{d t}+C_{5 A} \frac{d \delta_{A}}{d t}+C_{5 r} \frac{d r_{f}}{d t}+C_{5 c}
$$

$$
+\frac{1}{\frac{\left(C_{p}\right)_{T_{f}}}{T_{f}}+\frac{d\left(C_{p}\right)_{T_{f}}}{d T_{f}}}
$$

$C_{5 T}=C_{51}\left[\frac{1}{(k)_{T_{f}}} \frac{d(k)_{T_{f}}}{d T_{f}}-\frac{1}{\left(C_{p}\right)_{T_{f}}} \frac{d\left(C_{p}\right)_{T_{f}}}{d T_{f}}\right]$,

$$
\begin{aligned}
& \times\left(\frac{1}{T_{f}} \frac{d\left(C_{p}\right)_{T_{f}}}{d T_{f}}-\frac{\left(C_{p}\right)_{T_{f}}}{T_{f}^{2}}\right. \\
& \left.\left.+\frac{d^{2}\left(C_{p}\right)_{T_{f}}}{d T_{f}^{2}}\right)\right]
\end{aligned}
$$

$C_{5 r}=-\left(C_{52}+\frac{4}{3} C_{53}\right) \frac{2}{r_{f}}$,

$C_{5 c}=\left(C_{52}+\frac{1}{3} C_{53}\right) \frac{1}{\dot{E}_{e}} \frac{d \dot{E}_{e}}{d t}$,

$$
\frac{d \delta_{T}}{d t}=C_{8} \frac{d T_{f}}{d t}+C_{9} \frac{d r_{f}}{d t}+C_{10} \frac{d \delta_{A}}{d t}+C_{11},
$$

Substituting Eqs. A10 and A11 into A9 results in

$$
\frac{d C_{6}}{d t}=C_{6 T} \frac{d T_{f}}{d t}+C_{6 r} \frac{d r_{f}}{d t}+C_{6 A} \frac{d \delta_{A}}{d t},
$$

$$
\frac{d C_{7}}{d t}=C_{7 T} \frac{d T_{f}}{d t}+C_{7 r} \frac{d r_{f}}{d t},
$$

$C_{6 T}=-\left(\frac{1}{\operatorname{Le}_{A}} \frac{1}{\delta_{A}}+\left(1-\frac{1}{\mathrm{Le}_{A}}\right) \frac{2}{r_{f}}\right) C_{7 T}$

where

$$
\begin{aligned}
& -\frac{2(k)_{T_{f}}\left(T_{f}-T_{u}\right)}{\rho_{b} T_{b}} \\
& \times\left(\frac{1}{(k)_{T_{f}}} \frac{d(k)_{T_{f}}}{d T_{f}}+\frac{1}{T_{f}-T_{u}}\right),
\end{aligned}
$$

$$
\begin{aligned}
& C_{8}=\frac{-1}{2 C_{5} \delta_{T}+C_{6}}\left(C_{5 T} \delta_{T}^{2}+C_{6 T} \delta_{T}+C_{7 T}\right), \\
& C_{11}=\frac{-1}{2 C_{5} \delta_{T}+C_{6}} \delta_{T}^{2} C_{5 c}, \\
& C_{9}=\frac{-1}{2 C_{5} \delta_{T}+C_{6}}\left(C_{5 r} \delta_{T}^{2}+C_{6 r} \delta_{T}+C_{7 r}\right), \\
& C_{10}=\frac{-1}{2 C_{5} \delta_{T}+C_{6}}\left(C_{5 A} \delta_{T}^{2}+C_{6 A} \delta_{T}\right) .
\end{aligned}
$$

